FORMATION Formation emploi

Revue française de sciences sociales

104 | octobre-décembre 2008

Pêle-mêle

\title{
L'insertion des femmes artistes : entre obstacles culturels et choix rationnels
}

Integrating women artists: cultural obstacles and rational choices

Eingliederung von Künstlerinnen: zwischen kulturellen Zwängen und rationalen

Entscheidungen

La inserción de las artistas mujeres : entre obstáculos y elecciones racionales

\section{Magali Danner et Gilles Galodé}

\section{(Q) OpenEdition}

Journals

Édition électronique

URL : http://journals.openedition.org/formationemploi/1346

DOI : 10.4000/formationemploi.1346

ISSN : 2107-0946

Éditeur

La Documentation française

Édition imprimée

Date de publication : 1 octobre 2008

Pagination : $37-52$

ISSN : 0759-6340

Référence électronique

Magali Danner et Gilles Galodé, «L'insertion des femmes artistes : entre obstacles culturels et choix rationnels », Formation emploi [En ligne], 104 I octobre-décembre 2008, mis en ligne le 01 octobre 2010, consulté le 30 octobre 2020. URL : http://journals.openedition.org/formationemploi/1346 ; DOI : https://doi.org/10.4000/formationemploi.1346 
transposée à des carrières qui ne se déroulent pas dans le cadre d'une organisation. Ainsi, les professions indépendantes n'offrent pas en soi de progression verticale suivant une logique hiérarchique mais proposent en revanche une progression similaire suivant une logique de renommée. Dans les professions artistiques, en particulier, «(...) rien de plus finement hiérarchisant que la cotation par la réputation individuelle » (Menger, 2003). L'ascension des femmes aux différents échelons de l'entreprise a donné lieu à une littérature scientifique abondante ; cependant, moins de travaux se sont intéressés à leur progression dans les niveaux de réputation. Pourtant, dans les secteurs liés à la création où on s'attend à trouver de nombreuses femmes, eu égard à leurs présupposés talents et dispositions naturelles, les plus grands chefs cuisiniers, artistes peintres, chorégraphes ou stylistes restent, dans l'imaginaire collectif, largement des figures plutôt masculines. Comment expliquer cette moindre présence des femmes artistes sur les devants de la scène?

S'agissant plus précisément des plasticiennes, qui sont au cœur de notre réflexion, de nombreuses études ont souligné leur difficulté à se maintenir sur un marché particulièrement sélectif. Leur travail, longtemps considéré comme un genre à part, voire mineur (Nochlin, 1994), est encore aujourd'hui sousreprésenté dans les lieux d'exposition (Quinby, 2003) ou dans les ouvrages de référence (Lamoureux, 2004). Quant à leurs progressions de carrière, elles restent bien moins avantageuses que celles des hommes, étant peu reconnues sur le plan professionnel et peu valorisées dans les circuits favorisant une ouverture vers le marché des œuvres (Pasquier, 1983).

Les recherches sur le genre, qui s'interrogent sur les modalités de reconnaissance de la femme artiste et observent son insertion plus laborieuse dans les réseaux de réputation, interpellent les normes dominantes. Historiquement forgées sur des modèles d'excellence masculins, ces normes artistiques, à l'aune desquelles les productions des femmes seraient évaluées, ne répondraient pas à un idéal méritocratique pourtant mis en avant par les experts (Buscatto 2000; Sorignet, 2004). Ces processus déterminant les chances de réussite tout au long de leur carrière, les femmes seraient finalement incitées à se détourner des professions artistiques où elles sont davantage sélectionnées que les hommes. Ainsi, non seulement les femmes artistes progresseraient moins dans la hiérarchie des cotations mais s'engageraient aussi moins souvent dans certaines professions dominées par l'élite masculine, les deux logiques étant intimement liées.

Si ces inégalités de parcours professionnel font l'objet des études sur l'égalité des chances dans l'emploi, peu de recherches se sont intéressées à la mise en place de ces différenciations dès le début de carrière. Les écoles d'art constituent, de ce point de vue, un terrain d'étude particulièrement adapté, et ce d'autant plus que, longtemps exclues de ces lieux de formation artistique (Heinich, 1993) $)^{2}$, les femmes y sont aujourd'hui majoritaires. Sélectionnant les étudiants dès l'entrée du cursus puis tout au long de leur scolarité, ces écoles constituent des filières qualifiantes. À l'issue du cycle long, le DNSEP (diplôme national supérieur d'expression plastique), équivalant à un diplôme de troisième cycle, certifie les compétences de l'étudiant en expression plastique et sa capacité à conduire un projet artistique.

Nous étudierons ici la façon dont les diplômés, dès la fin de leurs études, parviennent à valoriser professionnellement les compétences acquises en école d'art. Il s'agira en particulier de vérifier l'hypothèse d'une sélection anticipée des femmes diplômées pour le marché de l'art où elles semblent moins reconnues. Nous examinerons les facteurs objectifs qui pourraient inciter les femmes à valoriser différemment leur formation supérieure, par rapport aux hommes issus des mêmes écoles afin d'expliquer cette « construction» de la rareté chez la femme artiste.

\footnotetext{
2 En 1648, l'Académie royale de peinture et de sculpture est créée. Les élèves y suivent un long apprentissage auquel les femmes n'ont cependant aucun accès, et si certaines échappent à cette exclusion, c'est qu'il s'agit de filles ou femmes de peintres ou issues de riches familles aristocratiques. Ces femmes sont d'ailleurs limitées dans leur expression artistique à des domaines et des sujets considérés à l'époque comme mineurs (portraits, natures mortes, sujets religieux...). Après la Révolution, les femmes, considérées «en tout point différentes des hommes », doivent attendre 1880 pour que l'École des beaux-arts de Paris leur ouvre de nouveau les portes avec cependant un enseignement différencié jusqu'en 1897 (Heinich, 1993).
} 


\section{Encadré 1 \\ Méthode d'enquête}

Pour répondre à la question de l'insertion, cette recherche a bénéficié du soutien financier du ministère de la Culture. Elle a permis de suivre, sur trois années, une cohorte de 773 diplômés ayant obtenu leur DNSEP (diplôme national supérieur d'expression plastique), en juin 2003, représentant la quasi-totalité des sortants DNSEP des écoles nationales et régionales. Sur l'ensemble de cette population mère, 557 personnes ont pu être finalement retrouvées et interrogées à 18 mois (janvier 2005) et 36 mois (juin 2006) après l'obtention du diplôme.

Les thèmes du questionnaire portaient sur les caractéristiques sociodémographiques du diplômé, son cursus scolaire secondaire et en école d'art, sa situation professionnelle actuelle et la qualité de l'emploi occupé.

Une seconde enquête a été réalisée auprès des sortants de juin 2005, 472 personnes ont ainsi été interrogées, 18 mois et 36 mois après leur sortie de formation, sur des dimensions comparables à la précédente enquête (enquête téléphonique). Les caractéristiques générales de la population mère pour chaque génération suivie sont les suivantes:

Quelques caractéristiques de la population de diplômés, en \%

\begin{tabular}{|c|l|c|c|}
\hline \multicolumn{2}{|c|}{ Dimensions } & Enquête sortants 2003 & Enquête sortants 2005 \\
\hline \multirow{3}{*}{ Sociodémographiques } & Femmes & 54 & 59 \\
\cline { 2 - 4 } & Cadre supérieur & 27 & 32 \\
\hline \multirow{3}{*}{ Scolaires } & Option Art & 60 & 66 \\
\cline { 2 - 4 } & Option Communication & 20 & 16 \\
\cline { 2 - 4 } & Option Design & 20 & 18 \\
\hline
\end{tabular}

Les effectifs concernés par cette étude sur les diplômés du cycle long des écoles d'art sont faibles et n'autorisent pas des analyses statistiques complexes. Toutefois, s'agissant de données recueillies à partir d'une population exhaustive, les analyses restent représentatives et sont d'autant plus fiables que l'étude a été conduite sur deux générations successives de diplômés. Ceci autorise une comparaison qui conforte, malgré le manque d'effectifs, les conclusions sur les tendances observées.

\section{INÉGALITÉ D'INSERTION SUR LE MARCHÉ DU TRAVAIL}

L'enquête auprès de la génération sortante de 2005 montre que $66 \%$ de la promotion est en emploi 18 mois après la sortie d'école. Ces taux étaient quasiment équivalents pour la génération sortante de $2003(60 \%)$.

Dans une recherche conduite par Emmanuel Sulzer (1999) sur les sortants issus de plusieurs promotions de l'École des beaux-arts, il apparaissait que la prise en compte du cursus s'avérait indispensable pour anticiper les trajectoires professionnelles. En théorie, la durée de formation pour obtenir le DNSEP est de cinq années. Dès la troisième année, les étudiants doivent se spécialiser dans l'une des trois options proposées, à savoir : Art, Communication ou Design.

Comparés aux sortants de l'option Art, les diplômés des options Communication et surtout ceux issus de l'option Design ont des probabilités d'insertion nettement plus élevées : $60 \%$ pour les sortants de l'option Art, $70 \%$ pour les sortants de l'option Communication et $77 \%$ pour les sortants de l'option 
Tableau 1

Taux d'emploi des diplômés d'école d'Art, par genre, en \%, 18 et 36 mois après la fin des études

\begin{tabular}{|c|c|c|c|}
\hline & & Enquête 2005 & $\begin{array}{l}\text { Comparaison } \\
\text { Enquête } 2003\end{array}$ \\
\hline \multirow{2}{*}{ Genre } & Homme & $\begin{array}{l}18 \text { mois : } 68 \\
36 \text { mois : } 84\end{array}$ & $\begin{array}{l}18 \text { mois : } 66 \\
36 \text { mois : } 88\end{array}$ \\
\hline & Femme & $\begin{array}{l}18 \text { mois: } 62 \\
36 \text { mois : } 81\end{array}$ & $\begin{array}{l}18 \text { mois : } 55 \\
36 \text { mois : } 80\end{array}$ \\
\hline
\end{tabular}

Source : Magali Danner et Gilles Galodé, enquêtes sur les diplômés des écoles d'art (2005-2006 et 2007-2008).

Design. Dans l'articulation entre sphère de la connaissance et sphère de la production, les options traduisent d'une certaine manière un effort d'adaptation de la formation aux logiques de l'emploi (Sulzer, 1999). La spécialisation plus affichée des sortants de l'option Communication et Design les prépare sans doute mieux à occuper des emplois qui requièrent plutôt des savoir-faire techniques: alors que $44 \%$ des diplômés de l'option Art travaillent avec les musées, les expositions ou les galeries d'art, les diplômés de l'option Communication exercent plutôt dans l'édition, l'illustration, la presse $(21 \%)$ ou la publicité, le marketing (29\%) et les diplômés de l'option Design plutôt dans l'architecture, la décoration et l'agencement (42\%).

Plus précisément, les diplômés de l'option Art occupent principalement des emplois d'animation culturelle $(15 \%)$ et d'enseignement artistique (14\%) ou de plasticien $(21 \%)$. Bon nombre de diplômés ayant suivi ce cursus occupent aussi des emplois éloignés de la pratique artistique ou culturelle (22\%). Bien que les diplômés de l'option Communication soient aussi nombreux à exercer des métiers sans rapport avec la pratique artistique ou culturelle (20\%), près de la moitié (47\%) sont cependant graphistes ou infographistes. Ces deux emplois concernent $37 \%$ des sortants de l'option Design, que l'on retrouve aussi logiquement dans des emplois de designer $(25 \%)$.

Le rapport homme/femme dans ces options est équivalent. Toutefois, comme dans la plupart des emplois (Milewski, 2004), l'écart constant entre hommes et femmes témoigne d'une difficulté d'insertion significativement plus marquée pour ces dernières (Tableau 1), quelles que soient les options consi- dérées. À 18 mois, $68 \%$ des hommes et $62 \%$ des femmes issues de la génération 2005 sont en situation d'emploi. Bien que ces écarts soient réduits par rapport à la génération précédente (respectivement, $55 \%$ et $66 \%$ ), le taux d'insertion des femmes reste en-deçà de celui des hommes.

Les diplômées sont néanmoins les premières à occuper un emploi dès la sortie de leur formation mais le nombre de femmes entrant sur le marché du travail ralentit progressivement: quatre mois après l'obtention du diplôme, leur taux d'emploi se situe définitivement en-deçà de celui des hommes, ce constat se vérifiant pour les générations 2003 et 2005. Entre la sortie de l'école et la première période d'enquête à 18 mois, la quasi-totalité des personnes ( $90 \%$ en 2005 et $85 \%$ en 2003) ont occupé au moins un emploi. Beaucoup de diplômés ont connu plusieurs périodes d'activité avec la succession de deux emplois ( $29 \%$ en 2005 et $25 \%$ en 2003 ) ou plus ( $11 \%$ en 2005 et $15 \%$ en 2003). Sur ce point, aucune différence ne caractérise les hommes et les femmes.

L'insertion plus lente des femmes sur le marché du travail n'est pas spécifique aux études artistiques (Gréco, 2003). En revanche, les orientations professionnelles qu'elles assument à l'issue de leur formation supérieure en expression plastique méritent une attention particulière.

\section{LES DIPLÔMÉES PRÉFÈRENT LES EMPLOIS SALARIÉS}

S'agissant des personnes actives, les diplômés des écoles d'art s'orientent massivement vers les emplois 
Tableau 2

Statut des diplômés en emploi, par genre, 18 et 36 mois après la fin de leurs études

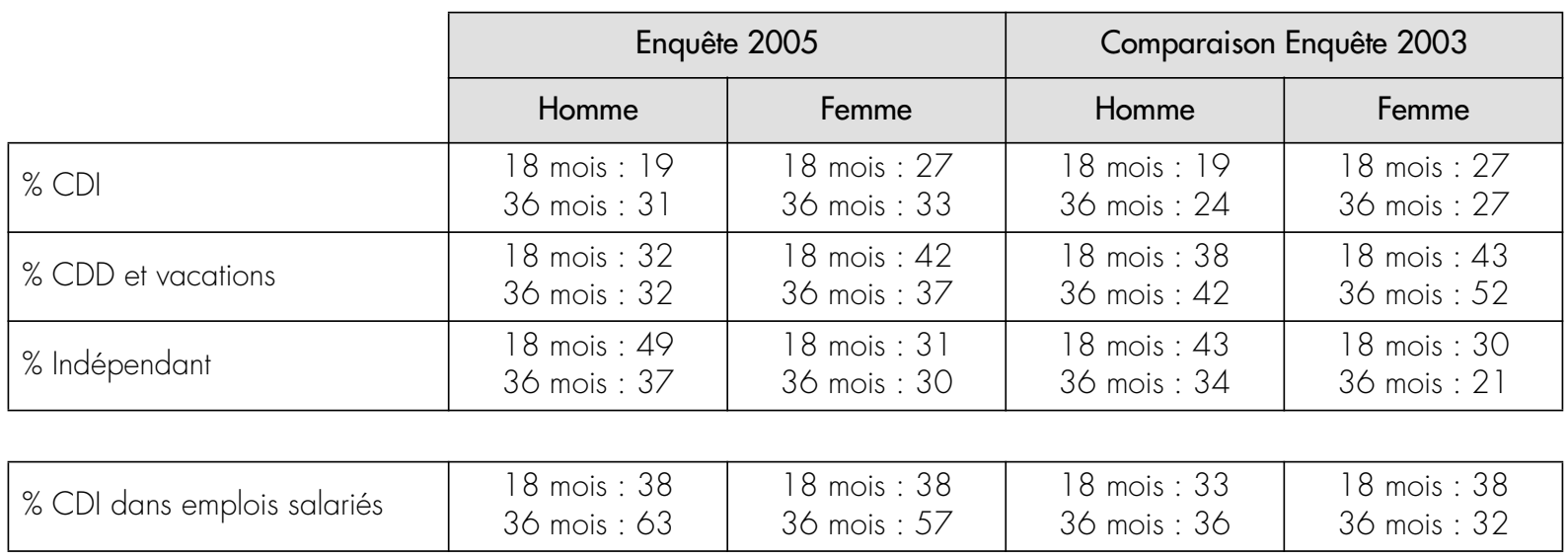

Sigles : CDI (contrat à durée indéterminée) ; CDD (contrat à durée déterminée).

Lecture : Sur l'ensemble de la promotion 2005, $19 \%$ des hommes sont en CDI 18 mois après la fin de leurs études et $31 \% 36$ mois après leurs études. Parmi les hommes en emplois salariés, $38 \%$ sont en CDI 18 mois après la fin de leurs études contre $63 \% 36$ mois après la fin de leurs études.

Source : Magali Danner et Gilles Galodé, enquêtes sur les diplômés des écoles d'art (2005-2006 et 2007-2008).

salariés, délaissant les professions indépendantes artistiques. Selon la génération de sortie, 35 à $39 \%$ d'entre elles travaillent en indépendant, 18 mois après l'obtention du diplôme.

Cependant, des écarts importants entre hommes et femmes existent et se maintiennent, avec une nette préférence des hommes pour ce choix de carrière : $31 \%$ des femmes diplômées exercent une profession indépendante artistique contre $49 \%$ des hommes (Tableau 2). Moins présentes dans les carrières indépendantes, elles sont plus souvent représentées dans les emplois salariés et, conjointement, plus nombreuses que les hommes à bénéficier de CDI (contrat à durée indéterminée), soit $26 \%$ contre $19 \%$ pour les hommes. Cependant, en ne considérant que les emplois salariés, la part des CDI est quasiment équivalente entre les hommes et les femmes ( $38 \%$ en 2005). Autrement dit, la différenciation homme/ femme, en début de carrière, repose sur une orientation professionnelle qui favorise en priorité les carrières artistiques indépendantes pour les hommes et les emplois salariés pour les femmes. Cela est également vrai pour la génération précédente, ce qui suggère une persistance de ces différences de positionnement professionnel. À 36 mois, le nombre de personnes exerçant en tant qu'indépendant diminue aussi bien pour les femmes que pour les hommes. Si, pour la génération 2005 (et contrairement à la génération 2003), les femmes paraissent se maintenir dans l'activité, elles restent toujours en deçà des effectifs masculins.

La promotion de diplômés de 2003, étudiée à 18 et 36 mois après l'obtention du diplôme, permet d'observer, au travers d'une table de mobilité, de quelle façon s'opère le changement de statut sur les trois premières années après la sortie de formation. L'étude de la mobilité professionnelle selon le genre montre que les tendances observées en amont semblent se renforcer dans le temps.

Les diplômés engagés dans des carrières artistiques indépendantes 18 mois après la sortie d'école conservent, pour la très grande majorité, ce statut un an plus tard. Toutefois, les femmes sont moins nombreuses à se maintenir dans l'activité : un certain nombre d'entre elles occupent désormais un emploi salarié en $\mathrm{CDI}$ ou suivent une formation.

Les emplois salariés sous CDI sont très stables avec $77 \%$ de personnes qui exercent sous le même statut d'une période à l'autre. Pour ces emplois, aucun écart significatif n'est à relever entre les hommes et les femmes. En revanche, les emplois salariés à durée déterminée suscitent une certaine mobilité. Alors qu'en moyenne le taux de maintien sous CDD 
(contrat à durée déterminée) est de $48 \%$, il est plus élevé chez les hommes ( $56 \%$ ) que chez les femmes (40\%). Celles-ci évoluent plus fréquemment vers des CDI ( $28 \%$ contre $6 \%$ pour les hommes) tandis que les hommes exercent plus souvent, un an après, en tant qu'artiste indépendant ( $17 \%$ contre $9 \%$ pour les femmes).

C'est précisément l'inverse qui se produit pour les diplômés en formation à 18 mois. Si le tiers d'entre eux sont inactifs à 36 mois, les autres se répartissent de façon inégale entre les différentes situations professionnelles avec davantage de femmes qui s'orientent vers les professions artistiques indépendantes ( $17 \%$ contre $8 \%$ d'hommes) et davantage d'hommes qui travaillent sous CDI (25\% contre $7 \%$ de femmes). Enfin, les femmes sans emploi restent plus longtemps en situation d'inactivité que les hommes, sont moins nombreuses à privilégier un changement de situation au profit des carrières artistiques indépendantes et sont beaucoup plus souvent engagées dans des formations complémentaires à leur cursus.

Les travaux de la DEP (2004) et la plupart des recherches conduites en relation avec les mondes artistiques, qu'il s'agisse de la danse, du chant, des lettres ou encore des arts, ont souligné l'inégale représentation des hommes et des femmes selon les activités considérées dans chaque champ. La recherche sur les diplômés du cycle long des écoles d'art vise à étudier comment, dès le début de carrière, ces différenciations s'instaurent et quelles stratégies guident les uns et les autres vers des choix professionnels au final relativement typés. L'étude de la relation formation-emploi de diplômés issus d'une formation artistique de niveau élevé offre une opportunité d'autant plus intéressante que ces diplômés sont tous préparés à entrer dans des carrières artistiques; dès lors, en quoi les spécificités individuelles, et notamment le genre, peuvent-elles influencer les orientations professionnelles?

\section{SALARIÉ OU INDÉPENDANT : QUELS RISQUES POUR QUELS BÉNÉFICES?}

Le poids de l'histoire, évoqué en introduction, joue certainement sur les stratégies d'orientation puisque, pendant des générations, rares étaient les femmes qui pouvaient exercer en tant que plasticiennes et exceptionnelles étaient celles qui parvenaient à faire reconnaître leurs talents artistiques (Heinich, 1993). Ainsi, l'exclusion des femmes du monde de l'art et leur faible valorisation, encore aujourd'hui, dans les lieux d'exposition ou les ouvrages de références, éclairent

Tableau 3

Avantages apportés par le statut professionnel (diplômés 2005), 18 mois après la fin des études

\begin{tabular}{|c|l|c|c|c|}
\cline { 3 - 4 } \multicolumn{2}{c|}{} & CDI & CDD & Indépendant \\
\hline \multirow{3}{*}{ Sécurité } & Estiment être dans une situation stable & $66 \%$ & $31 \%$ & $34 \%$ \\
\cline { 2 - 5 } & Estiment avoir des revenus réguliers & $99 \%$ & $77 \%$ & $15 \%$ \\
\cline { 2 - 5 } & Personnes qui déclarent exercer à temps plein & $62 \%$ & $38 \%$ & $59 \%$ \\
\hline \multirow{3}{*}{\begin{tabular}{c} 
Qualité \\
\cline { 2 - 4 }
\end{tabular}} & $\begin{array}{l}\text { Estiment utiliser les compétences acquises en écoles } \\
\text { R'art }\end{array}$ & $62 \%$ & $66 \%$ & $92 \%$ \\
\cline { 2 - 5 } $\begin{array}{c}\text { Revenu } \\
\text { mensuel net } \\
\text { moyen }\end{array}$ & Part moyenne de la production artistique dans l'emploi & $27 \%$ & $36 \%$ & $61 \%$ \\
\cline { 2 - 5 } & Temps partiel & $1291 €$ & $683 €$ & $427 €$ \\
\cline { 2 - 5 } & $\%$ au-dessus du SMIC (980€) & $1334 €$ & $636 €$ & $921 €$ \\
\hline
\end{tabular}

Sigles : CDI : contrat à durée indéterminée. CDD : contrat à durée déterminée. SMIC : salaire minimum interprofessionnel de croissance. Note de lecture : $66 \%$ des diplômés en CDI estiment être dans une situation stable, pour $31 \%$ des personnes occupant un emploi salarié de type CDD et $34 \%$ des personnes exerçant en tant qu'artiste indépendant.

Source : Magali Danner et Gilles Galodé, enquêtes sur les diplômés des écoles d'art (2005-2006 et 2007-2008). 
ce processus d'auto-sélection. Toutefois, ce seul argument n'est pas convaincant. Si l'on admet l'hypothèse d'un arbitrage coût-bénéfice-risque, il faut aussi que les femmes trouvent un certain bénéfice à s'orienter vers les emplois salariés. Or, ceux-ci semblent notamment offrir de meilleures conditions de rémunération (Tableau 3). Pour la génération 2005, quasiment toutes les personnes en emploi salarié, et surtout sous CDI, perçoivent des revenus réguliers qui sont bien plus élevés que ceux des indépendants : en moyenne, ils s'élèvent à $1333 €$ mensuel (pour les temps pleins) et plus de $60 \%$ des salariés sous contrat à durée indéterminée gagneraient plus du SMIC (salaire minimum, soit 980 euros). En comparaison, le profil des indépendants paraît nettement moins avantageux: quatre indépendants sur cinq ne perçoivent pas de revenus réguliers. Le revenu moyen de ceux qui exercent à temps plein se situe en dessous de $1000 €$ par mois et seuls $32 \%$ des indépendants ont des revenus supérieurs au SMIC. Vivre de son art est donc difficile et peu nombreux sont les indépendants qui s'estiment être, 18 mois après leur sortie de formation, dans une situation stable $(34 \%$ contre $66 \%$ pour les personnes en CDI). À 36 mois, la situation évolue favorablement pour la majorité des personnes en emploi salarié : $95 \%$ des personnes en CDI et $77 \%$ des personnes en CDD déclarent percevoir des revenus réguliers et, respectivement, $78 \%$ et $31 \%$ s'estiment être dans une situation stable. Pour les indépendants, la situation s'améliore aussi, bien qu'elle reste toujours bien moins favorable que celle des personnes en emploi salarié avec $21 \%$ qui estiment bénéficier de revenus réguliers et $42 \%$ qui jugent leur situation stable. Le revenu mensuel moyen net des indépendants est de $1136 €$ (avec cependant de fortes variations) tandis que celui des personnes en CDI est de $1219 €(964 €$ pour les personnes en $\mathrm{CDD}$ ).

En revanche, si l'entrée dans les emplois salariés présente un moindre risque financier, elle s'opère au détriment de la qualité de l'emploi par rapport à la formation suivie. Ainsi, $92 \%$ (95\% à 36 mois) des indépendants déclarent utiliser les compétences acquises au cours de leur formation en école d'art, contre $62 \%$ (68\% à 36 mois), pour les personnes en CDI (respectivement $66 \%$ et $63 \%$ pour les personnes en CDD). Plus précisément, la part de production artistique dans l'ensemble des tâches est de $61 \%$ du temps de travail d'un indépendant contre $27 \%$ pour un salarié. À 36 mois, la comparaison des tâches principales exercées dans l'emploi montre bien des positionnements différents. La production artistique représente $77 \%$ du temps de travail mensuel contre $33 \%$ pour les emplois salariés.

La situation des salariés à 18 mois, et qui le sont toujours à 36 mois, a évolué vers plus de stabilité et une meilleure correspondance dans la relation formation-emploi : $90 \%$ perçoivent des revenus réguliers, $56 \%$ jugent leur situation stable et $67 \%$ travaillent à temps complet. Ils sont désormais $71 \%$ à déclarer utiliser leurs compétences artistiques acquises en école d'art. Ils sont cependant $44 \%$ à envisager de changer d'emploi, essentiellement pour bénéficier de meilleurs revenus (49\%) mais aussi en raison d'une certaine insatisfaction quant à la qualité de l'emploi (faible épanouissement : $21 \%$ ou faible utilisation des compétences artistiques : $26 \%$ ). Cette recherche d'adéquation dans la relation formation-emploi explique sans doute que $12 \%$ des personnes salariées à 18 mois sont devenues indépendantes un an et demi plus tard. Si l'épanouissement professionnel est une donnée essentielle dans le travail, elle ne suffit pas. Ceux qui se déclaraient indépendants à 18 mois sont nombreux à cesser cette activité pour privilégier des emplois salariés (25\%), notamment en CDI (16\%) ou à se lancer dans la recherche d'emploi (5\%). Le taux de stabilité ( $70 \%$ ) reste néanmoins assez élevé compte tenu des difficultés financières auxquelles sont confrontés les diplômés. En effet, parmi les indépendants qui se sont maintenus dans l'activité lors de la seconde enquête, $77 \%$ estiment être dans une situation précaire tout en travaillant cependant, pour la très grande majorité, à temps complet (68\%). Ils sont pourtant moins nombreux que les salariés à envisager de changer de situation professionnelle $(36 \%)$, uniquement en raison de l'insuffisance des revenus $(88 \%)$.

Les statuts professionnels n'offrent par conséquent pas les mêmes garanties. Cette étude comparative sur l'appréciation de l'emploi entre salariés et indépendants montre que la décision de devenir indépendant se prend en partie à l'issue d'un arbitrage entre le risque financier lié au statut et les avantages en 
termes d'épanouissement artistique. Dans la mesure où l'orientation professionnelle diffère entre les hommes et les femmes, ces dernières étant plus souvent positionnées sur des emplois salariés, on s'attend à observer des différences qualitatives entre hommes et femmes dans l'emploi. Le marché du travail s'avère en général plus discriminant à leur égard mais ceci demeure particulièrement visible au niveau des emplois artistiques, comme en attestent les nombreuses recherches conduites sur le sujet. Dans un tel contexte, il s'agit donc de s'interroger sur la façon dont les diplômées des écoles d'art valorisent les compétences artistiques acquises au travers de leur formation longue dans les écoles d'art.

\section{FEMMES ARTISTES :} DES CHOIX PROFESSIONNELS INÉGALEMENT RISQUÉS

Une première différence qualitative se rapporte au sentiment de pouvoir valoriser ses études dans le cadre de son travail. Sur ce point, les diplômées déclarent moins utiliser leurs compétences artistiques que les hommes (respectivement $73 \%$ contre $80 \%$ ), surtout dans les emplois salariés en CDI (56\% contre $73 \%)$.

Les femmes sont nombreuses à avoir un CDD et les hommes à exercer en tant qu'indépendants. Ces deux situations professionnelles étant généralement plus aléatoires que les emplois en CDI, au final, les femmes sont aussi nombreuses que les hommes à juger leur situation précaire $(68 \%)$ mais moins nombreuses que ceux-ci à avoir le sentiment de bénéficier de revenus réguliers (respectivement $41 \%$ contre $48 \%$ ). Les écarts de salaires ne paraissent pourtant pas significatifs, malgré des rémunérations mensuelles en moyenne légèrement moins importantes pour les femmes ( $931 €$ contre $1008 €$ pour les hommes).

Si pour les emplois salariés en CDI, ces différences peu significatives de salaires pouvaient s'expliquer par la représentation plus importante des femmes au niveau du temps partiel, cet argument ne se vérifie plus au niveau des professions indépendantes et des emplois salariés en CDD : bien que travaillant moins souvent que les hommes à temps partiel, elles sont moins bien rémunérées (Tableau 4).

L'analyse de régression linéaire multivariée permet de prendre en compte d'autres dimensions individuelles tout en contrôlant le facteur " temps partiel ». Précédemment, nous avions montré que les options suivies en école d'art influençaient les carrières avec une part importante de designers et graphistes parmi les diplômés des options Communication et Design. Or, les revenus sont considérablement plus élevés dans ces métiers (1 $100 €$ en moyenne pour un graphiste, $1276 €$ pour un designer) que dans ceux de l'animation $(679 €)$ ou de la création $(535 €)$,

Tableau 4

Situation professionnelle par genre (diplômés 2005), 18 mois après la fin des études

\begin{tabular}{|c|c|c|c|c|}
\hline & \multicolumn{2}{|c|}{ Revenus mensuels nets moyens } & \multicolumn{2}{|c|}{ Part du temps partiel } \\
\hline & Homme & Femme & Homme & Femme \\
\hline CDI & $\begin{array}{c}1109 \\
(n=25)\end{array}$ & $\begin{array}{c}1045 \\
(n=41)\end{array}$ & $31 \%$ & $42 \%$ \\
\hline CDD & $\begin{array}{c}934 \\
(n=40)\end{array}$ & $\begin{array}{c}891 \\
(n=61)\end{array}$ & $63 \%$ & $60 \%$ \\
\hline Indépendant & $\begin{array}{c}918 \\
(n=42)\end{array}$ & $\begin{array}{c}802 \\
(n=37)\end{array}$ & $63 \%$ & $58 \%$ \\
\hline Moyenne & $\begin{array}{c}1008 \\
(n=107)\end{array}$ & $\begin{array}{c}931 \\
(n=139)\end{array}$ & $57 \%$ & $54 \%$ \\
\hline
\end{tabular}

Sigles : CDI : contrat à durée indéterminée. $\mathrm{CDD}$ : contrat à durée déterminée.

Source : Magali Danner et Gilles Galodé, enquêtes sur les diplômés des écoles d'art (2005-2006 et 2007-2008). 
voire de l'enseignement $(1016 €)$ qui concernent plutôt les sortants de l'option Art. Les revenus moyens sont donc très variables d'une option à l'autre: $1201 €$ pour les diplômés en Design, $1119 €$ pour les diplômés en Communication et seulement $781 €$ pour les diplômés en Art.

Le modèle ci-dessous ne concerne que les personnes en emploi salarié (Tableau 5). Ceci tient notamment à la prise en compte du temps partiel qui détermine fortement le niveau des revenus. Par ailleurs, cette étude porte sur les premiers mois qui suivent l'entrée sur le marché du travail; dès lors, les variables sociales et de cursus introduites ont un certain impact sur l'explication des différences individuelles de revenus, les variables de carrière n'ayant pas eu le temps de se construire.

À temps partiel donné, les femmes ont significativement avantage à s'orienter vers les métiers de l'enseignement plutôt que vers les emplois de designer ou de graphiste. On sait aussi que les inégalités salariales sont moins fortes dans le public que dans le privé, ce qui explique sans doute en partie ce résultat. Toutefois, quel que soit le métier exercé, le salaire des femmes paraît davantage sujet à variation que celui des hommes. Ainsi, celles qui ont suivi un cursus professionnalisé gagnent significativement plus que les diplômées de l'option Art, plus généra- liste. Pour les hommes, cette dimension ne parait pas déterminer le niveau des revenus. De même, l'origine sociale n'est pas un facteur explicatif de la variance de revenus entre eux, alors qu'elle l'est pour les femmes. Sans pouvoir donner un contenu qualitatif à ces variables, il est évident que la détermination des salaires pour les femmes issues des écoles d'art subit davantage d'ajustement en fonction de leur origine sociale que pour les hommes.

L'étude des niveaux de rétribution par genre dans les professions indépendantes amène également quelques remarques. Au niveau de l'emploi, si les femmes renoncent plus souvent que les hommes à s'orienter vers les professions indépendantes artistiques, lorsqu'elles font cependant ce choix de carrière, elles occupent, de surcroît, bien moins souvent que les hommes le statut de plasticien (respectivement $19 \%$ et $26 \%$ ). Ainsi, en tant qu'indépendant elles exercent plutôt des professions de graphiste $(39 \%)$, designer $(10 \%)$ ou infographiste $(6 \%)$. En outre, ces professions indépendantes apportent aussi davantage de garanties financières que la profession de plasticien. En effet, les rétributions sont plus élevées, en moyenne $1097 €$ par mois contre moitié moins pour un plasticien (523€), mais aussi plus sûres, puisque $27 \%$ déclarent percevoir des revenus réguliers contre $3 \%$ des plasticiens.

Tableau 5

Régression linéaire sur les revenus, pour les salariés (diplômés 2005), 18 mois après la fin des études

\begin{tabular}{|l|l|c|c|}
\hline \multicolumn{1}{|c|}{ Référence } & \multicolumn{1}{c|}{$\begin{array}{c}\text { Modèle 1 } \\
\text { Variable muette }\end{array}$} & $\begin{array}{c}\text { Modèle 2 } \\
\text { revenu des hommes } \\
\text { (effectif }=60 \text { ) }\end{array}$ \\
\hline Constante & (effectif = 91) & 1536 \\
\hline Temps partiel & (Temps plein) & 1283 & -523 \\
\hline Diplôme Art & (Communication/Design) & -539 & $\mathrm{~ns}$ \\
\hline Autres métiers & (Designer/graphiste) & $\mathrm{ns}$ & -351 \\
\hline Enseignant & (Origine non cadre) & 279 & $\mathrm{~ns}$ \\
\hline Origine cadre & & 181 & $\mathrm{~ns}$ \\
\hline $\mathrm{R}^{2}$ & & $47 \%$ & $47 \%$ \\
\hline
\end{tabular}

Significativité au seuil de $p<0,1 ;$ is : non significatif

Note de lecture : Dans le modèle 1, on cherche à expliquer ce qui justifie les différences de revenus dans la sous-population des femmes (le modèle 2 concerne la sous-population des hommes). Ce modèle 1 observe que les femmes qui travaillent à temps partiel gagnent significativement moins que celles qui travaillent à temps plein (en moyenne, $-539 €$ ). Les variables muettes sont les variables de référence (exemple : temps plein/temps partiel) à partir desquelles on étudie les conséquences de leur variation sur les revenus.

Source : Magali Danner et Gilles Galodé, enquêtes sur les diplômés des écoles d'art (2005-2006 et 2007-2008). 
Tableau 6

Revenus mensuels nets moyens par genre, option et temps travaillé, pour les indépendants (diplômés 2005), 18 mois après la fin des études

\begin{tabular}{|l|l|c|c|c|c|c|c|}
\cline { 3 - 8 } \multicolumn{2}{c|}{} & \multicolumn{4}{c|}{ Femmes } & \multicolumn{3}{c|}{ Hommes } \\
\cline { 3 - 8 } \multicolumn{2}{c|}{} & $\begin{array}{c}\text { Temps } \\
\text { plein }\end{array}$ & $\begin{array}{c}\text { Temps } \\
\text { partiel }\end{array}$ & Moyenne & $\begin{array}{c}\text { Temps } \\
\text { plein }\end{array}$ & $\begin{array}{c}\text { Temps } \\
\text { partiel }\end{array}$ & Moyenne \\
\hline \multirow{2}{*}{$\begin{array}{l}\text { Design/ } \\
\text { Communication }\end{array}$} & Rétributions & $1001 €$ & $958 €$ & $988 €$ & $2073 €$ & $900 €$ & $1116 €$ \\
\cline { 2 - 8 } & Effectifs & 6 & 14 & 20 & 4 & 13 & 17 \\
\hline \multirow{2}{*}{ Art } & Rétributions & $355 €$ & $567 €$ & $434 €$ & $811 €$ & $723 €$ & $771 €$ \\
\cline { 2 - 8 } & Effectifs & 10 & 6 & 16 & 13 & 11 & 24 \\
\hline
\end{tabular}

Note de lecture : 20 diplômées issues de l'option Design ou Communication exercent à temps plein la profession d'artiste indépendant. Elles perçoivent en moyenne un revenu équivalent à $988 €$.

Source : Magali Danner et Gilles Galodé, enquêtes sur les diplômés des écoles d'art (2005-2006 et 2007-2008).

La répartition des effectifs par profession n'autorise cependant pas la construction d'un modèle aussi complexe que celui présenté en amont. Le Tableau 6 montre cependant que les diplômés des options Art ont en général des revenus plus faibles que ceux des options professionnalisées, quels que soient les paramètres pris en considération (temps partiel, genre). On note aussi que, sur le marché des carrières artistiques indépendantes, le genre influe sur la détermination des revenus alors que cela n'apparaissait pas évident dans les emplois salariés. Ainsi, en moyenne, les hommes ayant suivi un cursus professionnalisé gagnent davantage (1 $116 €$ ) que les femmes (988 €). Les écarts sont encore plus nets pour les diplômées issues de l'option Art (771€ pour les hommes contre $434 €$ pour les femmes).

Parmi les facteurs susceptibles d'influencer le niveau d'un salaire, il y a donc l'origine sociale. Derrière cette variable se manifestent d'autres dimensions qui jouent généralement de façon positive sur la réussite professionnelle, comme le réseau relationnel, le capital culturel ou encore le niveau des ressources économiques apportées par la famille. Pour les diplômés qui envisagent la carrière d'artiste indépendant, le soutien familial est nécessairement important. En effet, il faut se constituer une clientèle et un réseau relationnel propre à consolider, par des «assortiments professionnels» sélectifs (Menger, 2003), une renommée. Par ailleurs, les apports financiers sont essentiels non seulement pour démarrer l'activité (achat de matériel, location d'atelier...) mais aussi pour vivre en attendant l'obtention d'une certaine notoriété. Pour ces raisons, les familles les plus modestes sont certainement moins incitées à soutenir les vocations artistiques de leurs enfants ; d'autant que ces familles, contrairement aux enfants de cadre ou d'artisan, n'ont pas le même rapport de proximité avec l'art ou les réseaux relationnels utiles pour s'imposer sur le marché artistique (Bourdieu et Darbel, 1966 ; Moulin et Quemin, 1993).

Le positionnement social initial ne joue cependant pas de la même façon selon le genre. Sur l'ensemble des diplômés DNSEP 2005, $25 \%$ des femmes de milieu cadre supérieur ou profession intellectuelle supérieure choisissent de devenir artiste indépendant, contre $16 \%$ de celles issues d'un milieu plus modeste. À titre de comparaison, pour les hommes, les taux sont de $33 \%$ pour les enfants de cadre comme pour ceux d'ouvrier/employé, témoignant que ce facteur de l'origine sociale est bien moins prégnant pour eux. Sans doute cette variable a-t-elle déjà contribué à sélectionner en amont les candidats pour des études en école d'art, mais en début de carrière son influence persiste pour les femmes alors qu'elle ne paraît pas avoir le même impact pour les hommes. Autrement dit, comme par le passé (Sauer, 1991), ce sont encore aujourd'hui plutôt les femmes issues des familles socialement les plus prestigieuses qui osent s'affirmer sur le marché du travail artistique. Par ailleurs, les femmes qui s'orientent vers les professions indépendantes ont de meilleurs revenus quand elles sont originaires d'un milieu aisé $(846 €)$ que les autres 
diplômées $(609 €)$ alors que pour les hommes, l'origine sociale n'influe pas sur les niveaux de rétributions (respectivement $941 €$ et $916 €$ ). Nos données quantitatives ne nous permettent pas de saisir comment cette variable exerce une influence sur les salaires ; cependant, elle parait jouer pour les diplômées à la fois sur la décision de s'affirmer en tant qu'artiste indépendant et sur la capacité à gagner plus rapidement sa vie grâce à cette activité.

L'étude des populations sans emploi permet d'approfondir ces éléments d'analyse. $64 \%$ des personnes en recherche d'emploi sont des femmes et $75 \%$ sont issues de l'option Art. Il existe donc, dès la sortie d'école, une population fragilisée à l'égard de l'emploi, à savoir les femmes issues de l'option généraliste Art. Ces personnes sont loin de rester inactives au niveau de la pratique artistique. En effet, les personnes en recherche d'emploi manifestent le souhait de se positionner à terme comme artiste plasticien (35\%), photographe, cinéaste $(13 \%)$ ou intervenant artistique (enseignement, médiation, animation : $14 \%$ ). La grande majorité n'envisage donc pas de travailler dans le secteur salarié mais davantage dans celui de la création indépendante, avec la volonté d'exister dans les lieux d'exposition comme les galeries, les musées ou les centres d'art (40\%). Par ailleurs, au-delà de ces projets, $92 \%$ de ces personnes ont conservé une activité artistique et $17 \%$ tirent ponctuellement des bénéfices de leur vente (en moyenne, $192 €$ par mois). La majorité (53\%) se dit insérée dans des réseaux professionnels porteurs et $66 \%$ envisagent de s'inscrire également à la Maison des Artistes ( $8 \%$ y sont déjà inscrits). Enfin, $31 \%$ disposent d'un atelier pour exercer leur activité artistique à laquelle ils consacrent en moyenne 102 heures par mois.

Que ce soit par leurs activités quotidiennes ou leurs ambitions professionnelles, les femmes restent au moins autant engagées que les hommes dans un projet de carrière artistique, plutôt orienté vers la pratique indépendante. Ainsi, loin d'être une fatalité, le chômage serait plutôt une alternative pour exister en tant qu'artiste, en attendant une certaine notoriété permettant de vivre de ses revenus.

Ces conclusions sont identiques à celles que nous pourrions tirer de l'enquête de 2003. Alors que les uns parviennent à tirer profit de la vente de leurs productions artistiques et s'assurent une lisibilité socioprofessionnelle, les autres restent dans l'ombre des aides institutionnelles ou familiales, tributaires d'un soutien financier extérieur, faute d'avoir trouvé dans l'immédiat un marché porteur pour leur activité. Vivant essentiellement du RMI (revenu minimum d'insertion : $42 \%$ ), des Assedic (22\%), d'emplois ponctuels ( $8 \%$ ) ou d'une aide des parents $(10 \%)$, ils disposent d'un apport financier de $581 €$ par mois en moyenne. C'est un peu moins que le revenu mensuel déclaré par les indépendants en exercice (646€ en moyenne, $361 €$ pour les plasticiens), avec la garantie de le percevoir quasi-régulièrement. Qu'il soit artiste indépendant ou en recherche d'emploi, chacun bénéficie d'un niveau de rémunération à peu près comparable et profite d'une liberté d'expression artistique ; les premiers accèdent cependant à un statut professionnel tandis que les seconds bénéficient de rentrées d'argent plus régulières.

\section{LES PLASTICIENNES RENTABILISENT-ELLES LEUR FORMATION SUPÉRIEURE ?}

Cette recherche montre que l'insertion des femmes diplômées des écoles d'art sur le marché du travail est moins aisée que celle des hommes. Quant à leur positionnement professionnel sur le marché du travail artistique, leur propension à envisager les carrières indépendantes est moindre que celle des hommes, et ce d'autant plus que l'on s'arrête sur la carrière de plasticienne. En outre, dans les emplois salariés comme dans les professions indépendantes, le choix du cursus et l'origine sociale semblent jouer plus fortement sur la détermination des niveaux de revenus que pour les hommes.

Nous rejoignons la position de Jacques Commaille (1992) qui invite à dépasser l'idée que les femmes seraient des victimes passives d'une discrimination sur le marché du travail. Elles peuvent en effet opter pour des orientations professionnelles qui servent leurs intérêts. Les analyses sociologiques et historiques évoquées précédemment constatent que, sur le 


\section{Tableau 7 \\ Exercice d'activités artistiques dans un cadre extraprofessionnel, par genre, 18 mois après la fin des études}

\begin{tabular}{|l|c|c|c|c|}
\cline { 2 - 4 } \multicolumn{1}{c|}{} & \multicolumn{2}{c|}{ Enquête 2005} & \multicolumn{2}{c|}{ Enquête 2003} \\
\cline { 2 - 5 } \multicolumn{1}{c|}{} & Homme & Femme & Homme & Femme \\
\hline Exerce une pratique amateur & $80 \%$ & $84 \%$ & $87 \%$ & $86 \%$ \\
\hline Temps consacré à cette activité par mois & $79 \mathrm{~h}$ & $62 \mathrm{~h}$ & - & - \\
\hline Réseau professionnel & $58 \%$ & $43 \%$ & $52 \%$ & $50 \%$ \\
\hline Possession d'un atelier & $39 \%$ & $24 \%$ & $39 \%$ & $33 \%$ \\
\hline Perçoit des rétributions & $22 \%$ & $18 \%$ & $31 \%$ & $22 \%$ \\
\hline Montant moyen des rétributions & $308 €$ & $198 €$ & - & - \\
\hline
\end{tabular}

Note de lecture : $80 \%$ des hommes exercent une pratique artistique en amateur (génération 2005). Cela concerne $84 \%$ de femmes. Source : Magali Danner et Gilles Galodé, enquêtes sur les diplômés des écoles d'art (2005-2006 et 2007-2008).

marché de l'art, les plasticiennes rencontrent plus de difficultés que les hommes à faire valoir leur talent ${ }^{3}$. Dans ce contexte de sélectivité forte, les femmes diplômées des écoles d'art vont vers les professions qui leur assurent non seulement de meilleures chances de survie sur le marché du travail mais aussi des revenus sensiblement équivalents à ceux des hommes, comme les métiers de l'enseignement ou les activités de graphisme et design. Que ce soit donc dans les emplois salariés ou les emplois indépendants, les femmes diplômées des écoles d'art se positionnent plutôt sur des emplois qui offrent une certaine garantie financière.

Ces emplois ne permettent cependant pas d'exprimer aussi fréquemment que les hommes leurs compétences artistiques, et d'ailleurs la moindre affirmation de la femme artiste diplômée du cycle long des écoles d'art se confirme au niveau des pratiques extra-professionnelles (Tableau 7). En effet, même

${ }^{3}$ Ceci est loin d'être spécifique au métier de plasticien. Une étude de Pierre-Emmanuel Sorignet (2004) conclut que dans le domaine de la danse, les femmes sont plus soumises que les hommes à la concurrence du nombre et aux canons de l'excellence, ce qui réduit leur probabilité d'être retenues lors des auditions. Marie Buscatto (2000) observe, elle aussi, que dans le domaine musical, les femmes ne subissent pas les mêmes pressions sociales que les hommes. Enfin, Christine Mennesson (2007) montre qu'être sportive professionnelle dans un sport masculin soumet le corps à une double épreuve, à savoir la maitrise de techniques corporelles masculines et la mise en valeur de certains stéréotypes propres au genre féminin (féminité, esthétisme, grâce...), pour à la fois convaincre de ses compétences professionnelles mais aussi répondre aux attentes sociales à leur égard. dans la sphère privée, ces femmes diplômées sont moins nombreuses que les hommes à valoriser leurs compétences artistiques; elles sont en effet moins insérées dans un réseau relationnel susceptible de promouvoir leurs productions $(43 \%)$ que les hommes $(58 \%)$ et déclarant percevoir en moyenne des rétributions moindres (198€ contre $308 €$ pour les hommes). Elles disposent aussi moins souvent (24\%) que les hommes (39\%) d'un espace professionnel ou d'un atelier, pour exercer leurs activités et consacrent en moyenne moins de temps que les hommes à ces pratiques (respectivement $62 \mathrm{~h}$ par mois contre $7 \mathrm{~h}$ par mois).

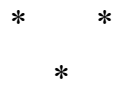

Les nouveaux modes de régulation du marché et la persistance d'une économie jugée incertaine ont conduit les artistes à rompre avec un modèle hérité du XIXe siècle (Moulin, 1992). Devant ces évolutions, les processus de certification mis en place dans les écoles d'art encouragent les jeunes diplômés à valoriser leur titre là où ils peuvent se monnayer, à savoir les emplois salariés. Se faisant, ces diplômés évoluent davantage vers le statut de «travailleur culturel » (Menger, 2003) plutôt que vers celui, plus marginal, de créateur indépendant.

$\mathrm{Au}$ travers de cette recherche, nous avons voulu montrer que les femmes valorisaient différemment 
leur formation supérieure en art plastique par rapport aux hommes issus des mêmes écoles. Comme dans bien des secteurs de l'économie, les femmes artistes rencontrent plus de difficultés que les hommes à s'insérer, alors même qu'elles ont suivi une formation de haut niveau laissant penser à une certaine maîtrise dans leur champ de compétences (Pasquier, 1983). Sur le marché spécifique de la création artistique, elles sont aussi moins présentes que leurs homologues masculins, que soient considérés les emplois salariés, les carrières artistiques indépendantes ou plus simplement les pratiques artistiques amateurs. Les femmes sont, au demeurant, d'autant plus incitées à renoncer à valoriser leur formation artistique dans des professions indépendantes qu'elles sont issues d'un milieu social distant de cet univers culturel, à moins de choisir une orientation qui les positionne, non pas sur le marché de l'art, mais sur celui de la promotion des biens de consommation (publicité, marketing, édition, web design, design...). S'agissant de productions commanditées en lien avec le secteur industriel ou commercial, c'est d'ailleurs dans les emplois salariés qu'elles peuvent espérer retirer les plus grands bénéfices en termes de sécurité de l'emploi et de niveau de rémunération. Si les recherches sur le champ artistique dénoncent des obstacles qui pourraient s'apparenter au «plafond de verre » et qui empêchent les femmes d'accéder au rang des artistes les plus reconnus, notre étude montre que, dès leur début de carrière, elles se heurtent aussi à un « mur de verre ». En effet, les femmes sont peu nombreuses à exercer en tant que plasticiennes. La profession ne leur est certes pas refusée explicitement mais il semble que leurs conditions d'exercice étant peu favorables comparativement à celles des hommes, elles renoncent plus facilement à ce type de carrière. Les analyses sur les personnes à la recherche d'un emploi invitent cependant à pondérer ces propos. Les femmes, nombreuses dans cette situation d'attente, ne paraissent pas avoir abandonné le projet de devenir artiste indépendant mais préfèrent rester dans l'ombre des aides institutionnelles plutôt que de se risquer à s'affirmer professionnellement. Cette moindre appétence pour la compétition sur le marché de l'art se traduit aussi au niveau des pratiques amateurs, où les femmes paraissent moins visibles que les hommes. Enfin, cette recherche ne s'inscrit pas dans une perspective longi- tudinale qui aurait pu étudier d'éventuels retours à l'activité sur une période d'observation plus longue. Sur la base de ces considérations, rien ne nous permet d'affirmer que les femmes ont définitivement renoncé aux carrières de plasticiennes. Cela étant, dès la fin de leurs études, elles paraissent moins souvent envisager ce type d'orientation professionnelle comparativement aux hommes. Les conclusions de cette recherche vont dans le sens d'autres travaux sociologiques comme ceux de Marie Buscatto (2000) ou encore ceux de Gonnard et Lebovici (2007) selon lesquels les femmes artistes ne parviennent pas aussi bien que les hommes à valoriser leurs compétences sur le marché de l'emploi artistique. Ces travaux de recherche convergent tous autour d'une question que posait déjà, en 1971, Linda Nochlin, historienne de l'art, dans la revue Artnews : "Pourquoi n'y a-t-il pas eu de grandes femmes artistes? 》 Est-ce à dire, poursuit-elle, comme le laissent insidieusement suggérer les polémiques autour de la question féministe qu' ' il n'y a aucune grande femme artiste parce que les femmes sont incapables de grandeur»? (Nochlin, 1988).

Nombre de sociologues mettent en avant le poids de l'histoire, qui a largement structuré le marché de l'art tout au long des siècles. Dans nos sociétés modernes, l'activité artistique est souvent perçue ou analysée en termes de talent, de don ou de vocation (Ivens, 2002). Or, ces représentations occultent les conflits qui structurent ce champ de production. Un champ s'organise dès lors qu'un corps de spécialistes s'arroge le pouvoir de l'authentification, faisant admettre comme légitime une certaine pratique sociale (Weber, 1996). Pierre Bourdieu (1977) a montré combien ces luttes de pouvoir déterminaient la valeur d'une œuvre. Le marché de l'art est empreint de ces conflits visant à instaurer un ordre symbolique autour duquel se construit la légitimité d'une œuvre. Or, les professions de la critique et de l'expertise des œuvres sont encore et toujours des professions dans lesquelles les hommes sont largement surreprésentés. De ce point de vue, les femmes qui osent s'affirmer en tant qu'artiste peinent davantage que leurs homologues masculins à faire valoir leur talent sur la scène publique (Mac Call, 1978; Pasquier, 1983). Parlant des musiciens de Jazz, Marie Buscatto (2000) résume bien les limites culturelles 
qui s'imposent aux femmes artistes: "Des conventions sociales, langagières et musicales "masculines" guident les relations de travail, rendant difficiles l'accès et le maintien des femmes chanteuses sur le marché de l'emploi musical du jazz. Des stéréotypes féminins, partagés par l'essentiel des musiciens rencontrés, rendent les femmes chanteuses peu "employables" et les enferment dans des positions musicales peu légitimes. »'

De même, Lourdes Mendez (1991), à propos des plasticiennes issues des beaux-arts, montre combien les mythes culturels construits autour de l'artiste conduisent ces dernières à un réel conflit identitaire. Étant à la fois femmes et artistes, elles aspirent à une reconnaissance de leur identité sociale d'artiste tout en cherchant à assumer une identité sexuelle qui non seulement les dessert sur le plan professionnel au niveau de l'ouverture sur le marché des œuvres mais les amène aussi à entrer en contradiction avec ce que les rapports sociaux entre les sexes exigent d'elles. Les femmes artistes doivent donc résoudre la tension entre taire sur le marché de l'art une identité féminine dévalorisée et jouer le rôle social qui incombe aux femmes dans les rapports quotidiens. La recherche conduite ici sur les sortants DNSEP (diplôme national supérieur d'expression plastique) de deux générations (2003 et 2005) rappelle combien ces obstacles culturels qui se dressent devant les jeunes diplômées des écoles d'art continuent aujourd'hui d'exercer une influence sur leurs choix d'orientation professionnelle en les incitant à renoncer plus facilement que les hommes aux carrières d'artiste indépendant, et plus particulièrement de plasticiennes.

\section{Bibliographie}

Bourdieu P. (1977), « La production de la croyance : contribution à une économie des biens symboliques », Actes de la Recherche en Sciences sociales, 13, pp. 13-45.

Bourdieu P. (1979), La distinction, Ed. de Minuit, Paris.

Bourdieu P. et Darbel A. (1966), L'amour de l'art, les musées et leur public, Ed. de Minuit, Paris.

Bureau International du Travail (BIT) (1997), La promotion des femmes aux postes de direction, Genève.

Buscatto M. (2000), « Chanteuse de Jazz n'est point un métier d'homme. L'accord imparfait entre voix et instrument », Revue française de sociologie, vol. 44, pp. 35-62.

Commaille J. (1992), Les stratégies des femmes. Travail, famille et politique, La Découverte, Paris.
DEP (2004), «Les établissements d'enseignement supérieur artistique et culturel dépendant du ministère de la Culture et de la Communication, effectifs et diplômes année scolaire 2003-2004», Les notes statistiques, $\mathrm{n}^{\circ} 11$ (décembre).

DEP (2004), «Les notes de l'Observatoire de l'emploi culturel», Série Données de cadrage. $\mathrm{n}^{\circ} 31$ et 30 (février), $\mathrm{n}^{\circ} 35$ et 36 (octobre).

Gonnard C., Lebovici I. (2007), Femmes artistes / artistes femmes, Paris, Hazan.

Gréco D. (2003), Les essentiels, Le chômage des femmes: portrait statistique, Noisy-le-Grand, L'Observatoire de l'ANPE.

Heinich N. (1993), Du peintre à l'artiste, artisans et académiciens à l'âge classique, Ed. de Minuit, Paris.

INSEE (1962), Recensement de la population. 
INSEE (2004), «Enquête emploi », in Insee Première, $\mathrm{n}^{\circ} 958$.

INSEE (2004), «Les salaires des dirigeantes de société en $2001 »$, Insee Première, nº 951.

Ivens M. (2002), Le peuple-artiste, cet être monstrueux, la communauté des pairs face à la communauté des génies, L'Harmattan, Paris.

Lamoureux E. (2004), «La question du genre dans les arts visuels : les femmes artistes mobilisées dans le féminisme au Québec », Actes de colloques, 26 et 27 novembre, Genre et militantisme, Lausanne.

Laufer J., Marry C., Maruani M. (dirs.) (2003), Le travail du genre. Les sciences sociales du travail à l'épreuve des différences de sexe, La Découverte, Coll. « Recherches ».

Laufer J. (2004), L'accès des femmes à la sphère de direction des entreprises : la construction du plafond de verre, Rapport de recherche commandité et financé par la DARES.

Lurol M. (2001), Le travail des femmes en France: trente ans d'évolution des problématiques en sociologie (1970-2000), Centre d'études de l'emploi, Document de travail $\mathrm{n}^{\circ} 7$.

Mac Call M. (1978), "The sociology of female artists », in Studies in symbolic Interaction, vol. 1, pp. 289-318.

Méndez L. (1991), « Ils voient des différences, elles n'en voient pas : des artistes femmes dans le champ de la production artistique $»$, Recherches féministes, vol. 4, n², pp. 61-74.

Menger P.-M. (2003), Portrait de l'artiste en travailleur, Coll. "Métamorphoses du capitalisme ", Seuil, Paris.
Mennesson C. (2007), «Les sportives professionnelles : travail du corps et division sexuée du travail », Cahiers du genre, $\mathrm{n}^{\circ} 42$, pp. 19-41.

Milewski F. (2004), « Travail des femmes et inégalités », Revue de l'OFCE, n 90.

Moulin R. (1992), L'artiste, l'institution et le marché, Flammarion, Paris.

Moulin R. et Quemin A. (1993), « La certification de la valeur de l'art. Experts et expertises ", Annales ESC, novembre-décembre, $\mathrm{n}^{\circ}$ 6, pp. 1421-1445.

Nochlin L. (1988), "Woman, Art and Power and Other Essays”, Westview Press, pp. 147-158.

Nochlin L. (1994), Why Have There Been No Great Women Artists. Writings about art, Englewood Cliffs, NJ. Prentice Hall, United-States.

Pasquier D. (1983), « Carrières de femmes : l'art et la manière », Sociologie du travail, $\mathrm{n}^{\circ} 4$, pp. 418-431.

Quinby D. (2003), Le collectif Femmes/Art à Paris dans les années 1970 : une contribution à l'étude du mouvement des femmes dans l'art, Thèse.

Sauer M. (1991), L'entrée des femmes à l'École des beaux-arts, 1880-1923, Coll. «Beaux-arts histoire», École Des Beaux-arts, Paris.

Sorignet P.-E. (2004), « Un processus de recrutement sur un marché du travail artistique : le cas de l'audition en danse contemporaine ", Genèses 57, pp. 6488 .

Sulzer E. (1999), Apprendre l'art. L'enseignement des arts plastiques et ses usages sociaux, 1973-1993, Paris, Presses Universitaires du Septentrion.

Weber M. (1996), Sociologie des religions, textes réunis par J.-P. Grossein, Paris. 


\title{
Résumé
}

\section{L'insertion des femmes artistes : entre obstacles culturels et choix rationnels}

\author{
Par Magali Danner et Gilles Galodé
}

Longtemps exclues des Académies et lieux d'exposition, les plasticiennes ont peiné à faire reconnaître leurs talents sur le marché de l'art. Aujourd'hui, les femmes peuvent suivre des formations supérieures en art plastique et sont majoritairement présentes dans les écoles d'art. Cette recherche s'intéresse aux diplômées de ces écoles et étudie leur insertion. Comme pour la plupart des études conduites sur les femmes artistes, elle montre combien les obstacles culturels continuent d'exercer une influence certaine sur les choix d'orientation professionnelle. Les diplômées des écoles d'art rencontrent ainsi des difficultés d'insertion plus importantes que les hommes mais renoncent aussi plus souvent à s'affirmer sur le marché de l'art, privilégiant des emplois leur offrant plus de garanties. Loin de considérer les diplômées comme des victimes passives d'une discrimination sur le marché du travail, cet article s'intéresse à la rationalité de leurs choix d'orientation professionnelle.

\section{Mots clés}

Insertion professionnelle, femme, art, enseignement supérieur, projet professionnel Journal of Economic Literature: J 16, Z 1 1, J 24 